\title{
Finite Difference Modeling of the Anisotropic Electric Fields generated by Stimulating Needles used for Catheter Placement
}

James Ch. Davis, Norman E. Anderson, Jason G. Ramirez, F. Kayser Enneking, and Mark W. Meisel

Abstract-The use of peripheral nerve blocks to control pain is an increasing practice. Many techniques include the use of stimulating needles to locate the nerve of interest. Though success rates are generally high, difficulties still exist. In certain deeper nerve blocks, two needles of different geometries are used in the procedure. A smaller needle first locates a nerve bundle, and then is withdrawn in favor of a second, larger needle used for injection. The distinct geometries of these needles are shown to generate different electric field distributions, and these differences may be responsible for failures of the second needle to elicit nerve stimulation when placed in the same location as the first. A 3-D finite difference method has been employed to numerically calculate the electric field distributions for a commercial set of stimulating needles.

Index Terms_-Anesthesiology, Electric fields, Finite difference methods 
Manuscript received November 18, 2005. This work was supported, in part, by the NSF through DMR-0305371 and by the University of Florida through the University Scholars and the Alumni Fellows Programs.

J. Ch. Davis, formally with the Department of Physics, University of Florida, Gainesville, FL 32611-8440 USA, is now with the Department of Physics, Ohio State University, Columbus, OH 43210-1117 USA, (email: lorentztrans@yahoo.com).

N. E. Anderson, formally with the Department of Physics, University of Florida, Gainesville, FL 32611-8440 USA, is now working at CH2M Hill, Gainesville, FL 32614-7009, USA.

J. G. Ramirez, formerly with the Department of Anesthesiology, University of Florida, Gainesville, FL 32611-8440, is now at the University of Colorado Health Sciences Center, Denver, CO 80262, USA, (email: jramirez@sdapc.com).

F. K. Enneking is with the Department of Anesthesiology, University of Florida, Gainesville, FL 32611-8440 USA, (email: kenneking@anest.ufl.edu).

M. W. Meisel is with the Department of Physics, University of Florida, Gainesville, FL 326118440 USA, (email: meisel@phys.ufl.edu).

Copyright (C) 2006 IEEE. Personal use of this material is permitted. However, permission to use this material for any other purposes must be obtained from the IEEE by sending an email to pubs-permissions@ieee.org. 


\section{INTRODUCTION}

Regional anesthetic techniques involve the use of local anesthetics to block neural impulse transmission from the peripheral nerves to the central nervous system. The application of local anesthetic can be done as a single bolus injection producing several hours of pain control. This type of regional anesthesia is termed peripheral nerve blockade (PNB). Local anesthetic medication can also be delivered over time through a catheter placed adjacent to nerves involved in pain transmission. This type of regional anesthesia is known as continuous peripheral nerve blockade (CPNB) and may result in pain control lasting several days. Both PNB and CPNB can provide excellent surgical anesthesia and postoperative analgesia for a variety of surgical procedures. Additionally, they have been shown to decrease the incidence of postoperative nausea and vomiting, reduce oral narcotic side effects, and improve sleep quality [1-3].

Placement of a PNB is typically done with a special needle, capable of transmitting an electric current from a power source to the neural structure. A neural target is located by applying a voltage to the needle, thereby generating a field of current splaying from the needle tip. When the field approaches, the nerve of interest is stimulated, as evidenced by a motor response (muscle contraction). Local anesthetic is then deposited through the needle in close proximity to the nerve. The needle is then removed, and over the course of several minutes, the local anesthetic acts to block nerve transmission. A similar procedure is followed for CPNB, in which a catheter is threaded through the needle system and left near the target nerve. Medication can be infused directly to the nerve of interest for a prolonged period of time through the catheter.

A typical procedure for placement of a CPNB system involves the use of two stimulating needles, a small one (Braun Stimuplex 21G) and a larger one (Braun Contiplex 18G), Figs. 1-2. The Stimuplex is first inserted near the neural target, and through visible muscle responses, the correct location and depth of neural tissue can be ascertained. The Stimuplex is preferable for initial location since the smaller gauge minimizes any potential tissue or vascular injury. Once 
the desired location is identified, the Stimuplex is withdrawn, and the Contiplex needle is introduced at the location and depth where optimal nerve stimulation occurred. After the Contiplex needle placement is adjusted to optimize stimulation, the local anesthetic is injected, and a nonstimulating catheter can be inserted through the Contiplex system.

This dual system is employed for deep perineural catheter placement locations such as the lumbar plexus and works well in a majority of catheter placements. Occasionally, a neural structure is easily identified with the Stimuplex needle, but lost upon placement of the Contiplex needle, despite meticulous technique. Previous studies have investigated the effects of shaft insulation and injectates on electric field distribution [4, 5], but not geometric variations between needles. Obvious design features, including diameter and area of insulating coverage, differ between the Stimuplex and Contiplex needles. It is reasonable to assume that differences are present in the stimulating field surrounding each needle tip. In an effort to better understand the process of $\mathrm{CPNB}$, we present numerically calculated stimulating fields present at the tip of the Stimuplex and Contiplex needle systems.

\section{MODEL}

The stimulating needles used in CPNB procedures locate the nerve of interest by causing an electric depolarization of the nerve, through an induced stimulus current, which elicits a motor response. The current density, $\vec{J}$, induced by the needle is related to the electric field $\vec{E}$ by Ohm's law,

$$
\vec{J}=\bar{\sigma} \vec{E}
$$

Here, $\overline{\bar{\sigma}}$ is the conductivity tensor associated with the medium. The uninsulated areas of the stimulating needles can be considered equipotential surfaces of potential $V$, and the electric field is then calculated from the electric potential using 


$$
\vec{E}=-\vec{\nabla} V
$$

The previous equations imply that the current density, and therefore the condition for nerve stimulation, can be determined from the electric potential. The problem then becomes a matter of solving Poisson's equation having a source region $g(x, y, z)$,

$$
\nabla^{2} V=g(x, y, z)
$$

with appropriate boundary conditions. These boundaries simply reflect the size and shape of the stimulating needle’s conductive and resistive surfaces.

Previous efforts showed that the grounded tissue/air boundary had no effect on the field distribution surrounding the needle tip, so the outer boundary condition is assumed continuous to simplify the problem [4]. The outer shaft of the needles will not substantially affect the electric potential due to the Teflon insulation. In the Contiplex needle, the inner surface is insulated with Teflon (see Fig. 2), so this surface does not contribute to the electric field profiles. Although the inside of the Stimuplex needle is bare, the inner metal surface is not in immediate contact with, i.e. it is sufficiently spatially remote from, the tissue being probed, so it is not considered in our analysis. Hence, the region at the tips of the needles, where the insulation is not present, is taken as the only source of electric field for the model calculations.

\section{METHODS}

Though exact solutions for very simple needle geometries have been found [6], Poisson's equation is not easily solved by analytical techniques for the complex geometries of the needles investigated. However, finite difference and finite element methods can be employed to numerically solve Poisson's equation [7]. The finite difference method was employed because it is easily adapted to 3-dimensions (3-D).

In order to probe the effect of needle geometry on nerve stimulation, a model consisting of a 
voltage source region $g(x, y, z)$, at the terminal end of the needle and a region of finite and constant conductivity surrounding the tip was used. Any differences seen in field profiles under these conditions will also be seen in a variable conductivity region such as the body [8]. In order to accurately model the needles, the uninsulated regions at the tips of the needles were measured under a microscope with the aid of an objective micrometer. Two of these magnified images are shown in Figs. 1 and 2.

A cubic mesh of size $(201)^{3}$ was generated, using MATLAB, with a mesh size of $0.03 \mathrm{~mm}$. As stated earlier, this problem reduces to solving Poisson's equation, which can be written in 3-D finite difference form as

$$
\begin{aligned}
V(i, j, k) & =\frac{1}{6}[V(i-1, j, k)+V(i+1, j, k)+V(i, j-1, k) \\
& \left.+V(i, j+1, k)+V(i, j, k-1)+V(i, j, k+1)-h^{3} g(i, j, k)\right] .
\end{aligned}
$$

Here $h$ is the mesh size and is used to represent the size of the source element for calculation. The source points are initialized to $0.5 \mathrm{~V}$ to provide the conditions of an average minimum current, $0.5 \mathrm{~mA}$, needed for stimulation, assuming a load resistance of $1 \mathrm{k} \Omega$ [9]. Although the maximum magnitude of applied voltage is, in clinical practice, user controlled, it is not tuned continuously while inserting the needles. Therefore, our results, obtained under constant voltage conditions, will scale linearly with respect to the field strength that is applied. The successiveover-relaxation method was used to iteratively solve for the potential throughout the mesh. In order to use this method, we define the following residual

$$
\begin{gathered}
R(i, j, k)=V(i-1, j, k)+V(i+1, j, k)+V(i, j-1, k)+V(i, j+1, k) \\
+V(i, j, k-1)+V(i, j, k+1)-6 V(i, j, k)-h^{3} g(i, j, k) .
\end{gathered}
$$

The electric potential can then be calculated at each mesh point using $R(i, j, k)$ for the $n^{\text {th }}$ iteration multiplied by a convergence factor $\omega$ to increase the convergence rate, i.e. 
TBME-00604-2005.R3

$$
V^{n+1}(i, j, k)=V^{n}(i, j, k)+\frac{\omega}{4} R^{n}(i, j, k) .
$$

Generally convergence factors between 1 and 2 are used; in this case a value of 1.2 produced an acceptable convergence rate for the simulation. As convergence is reached, $R(i, j, k)$ tends to zero, so the iterative process was stopped when the average value of $R(i, j, k)$ was less than $1.0 \mathrm{x}$ $10^{-5}$. This convergence limit leads to an average error in $V$ of less than $1 \%$. Using this convergence factor and limit, the simulations generally converged after 600 iterations.

Once $V$ was calculated, MATLAB's gradient calculation tool was utilized to determine the magnitude of the electric field distribution. Contour maps were generated for several planes around the needle tips and these maps were then used to depict the field profiles at a variety of positions and angles for the two needles.

\section{RESUlts}

Figures 3-6 summarize the results of the simulations for the Stimuplex and Contiplex needles. The points taken as equipotential areas (uninsulated conducting surfaces of the needles), are depicted by black points in each image. Each graph is a two-dimensional (2-D) slice of the computed 3-D field, and the insets display the orientation of this plane. Figures 3 and 4 show slices perpendicular to the shaft (z) axis for each needle, while Figs. 5 and 6 provide a different view. It should be noted for all graphs that the intensity of fields will scale linearly with source point voltage, and therefore the graphs will retain their shape under any physically meaningful change in applied tip voltage.

Figures 3 and 4 are a set of planes taken perpendicular to both needle's shafts at $0.3 \mathrm{~mm}$ above the needle point (a), exactly touching the point (b), and $0.3 \mathrm{~mm}$ below the point (c). In Fig. 3, the strongest field produced by the Stimuplex is found at the needle pinnacle, and along the $30^{\circ}$ uninsulated tip face. The Contiplex graphs (Fig. 4) have fewer points of constant voltage in this model, as a result of less uninsulated area on the needle. As illustrated in Fig. 4, the Contiplex 
also produces a strong field at the pinnacle, but, partially due to a shallower bevel angle, exhibits a less circularly homogeneous field compared to the Stimuplex.

Figure 5a shows the electric fields on a plane across the face of the Stimuplex needle. Conversely, Fig. 5b represents the field that would result across the same plane if the Stimuplex were replaced by the Contiplex at the same insertion angle and tip location. This scenario mimics

the ideal clinical case where the Stimuplex is inserted at a $30^{\circ}$ angle to the target nerve for initial location, and then replaced by the Contiplex at the same insertion angle and tip location. Even with this assumption of perfect clinical technique, the electric field is decreased considerably, and the areas of maximum field strength are limited to the upper region of the graph. These results directly mimic the case where nerve stimulation occurs readily with the Stimuplex needle, but is lost upon switching to the Contiplex.

The effects of rotations about the shaft (z) axis are shown in Fig. 6. Other than a $180^{\circ}$ needle rotation, Figs. 5 and 6 represent the same field area. Comparing the two graphs, however, reveals major shifts in the Stimuplex field distribution, and a less dramatic change in the Contiplex field. Additionally, along this plane a similar field is produced by each needle. For both needles, the field strength is decreased due to the z-axis rotation, even while maintaining the same tip location. Nerve stimulation in clinical practice could therefore be enhanced or lost entirely because of this type of rotation.

\section{DISCUSSION}

The results of our simulations demonstrate the inherent inhomogeneity of electric field profiles formed by the two needles investigated. Although the model is artificial in assuming a homogeneous conductive region within the body, incorporating a variable conductivity to reflect actual tissue variations should maintain or magnify the deviations in current density profiles [8]. The pinnacles of the needle tips both produce high fields in their immediate vicinity, but the 
distributions are otherwise dissimilar.

Since high field areas are seen around the entire tip face in both needles, total current induced through a nerve bundle will be highest when this face is parallel to the target nerve. For the Stimuplex, a $30^{\circ}$ beveled tip implies that this maximum stimulation occurs with the shaft inserted at this angle to the target. However, the Contiplex tip face makes a sharper angle with the shaft (taken as $15^{\circ}$ ), and thus maximum current through a target is achieved when the needle is inserted nearly parallel to the nerve. Since an insertion at such a sharp angle to the target nerve is unlikely, this difference in tip angle could explain a loss of stimulation upon needle replacement, even when the Contiplex tip is inserted exactly where stimulation previously occurred with the Stimuplex.

Similarly, the results show that a rotation about the shaft (z) axis could have a large effect on current through a nerve bundle. These rotations are difficult to eliminate entirely during insertion, so the technique of actively rotating needles in an exploratory fashion may be advisable to ascertain the optimal orientation. Alternatively, using a flat tip needle for initial nerve location could easily eliminate this dependence on rotation.

A two needle system in CPNB placement is designed to be less damaging by using a smaller needle for initial nerve location, and a larger one for injection and catheter placement. Inability to locate a target nerve by electric stimulation after switching needles can be attributed to the different current profiles generated by each needle. This loss of stimulation presently occurs in a small, but significant percentage of these procedures. Differences in the electric field profiles arising from the two needles could be reduced in several ways. For example, choosing needles with tip geometries that match one another would reduce occurrences of stimulation loss, thereby lessening the potential for tissue and nerve damage. In particular, needles with identical bevel angles would produce more similar fields. An overall increase in bevel angle will reduce field variation due to rotations, though an increased angle would not be practical with large gauge needles. On the other hand, simply insulating a larger portion of the tip face could have the same 
effect.

In new stimulating needle designs, the assumption that all insulated needles produce identical fields should not be taken. Even in procedures using a single needle to locate a nerve and inject anesthetic, the field profile is vital to ensuring proper placement. Successful anesthesia depends on the diffusion characteristic of an anesthetic once injected, not whether nerve stimulation has occurred. A needle design maximizing this correspondence between injectate spread and electric field distribution is ideal for any procedure. To this end, study of injectate dispersion in conjunction with electric characteristics would benefit future peripheral nerve blockading techniques. 


\section{REFERENCES}

[1] K. C. Nielsen and S. M. Steele, "Outcome after regional anaesthesia in the ambulatory setting--is it really worth it?,” Best Pract. Res. Clin. Anaesthesiol., vol. 16, pp. 145-157, 2002.

[2] B. M. Ilfeld, D. E. Esener, T. E. Morey, and F. K. Enneking, “Ambulatory perineural infusion: the patients' perspective,” Reg. Anesth. Pain Med. vol. 28, pp. 418-423, 2003.

[3] F. K. Enneking and B. M. Ilfeld, "Major surgery in the ambulatory environment: continuous catheters and home infusions,” Best Pract. Res. Clin. Anaesthesiol., vol. 16, pp. 285-294, 2002.

[4] G. Bashein, R. H. Haschke, and L. B. Ready, "Electrical nerve location: numerical and electrophoretic comparison of insulated vs uninsulated needles," Anesth. Analg., vol. 63, pp. 919-924, 1984.

[5] B. C. Tsui, A. Wagner, and B. Finucane, "Electrophysiologic effect of injectates on peripheral nerve stimulation,” Reg. Anesth. Pain Med., vol. 29, pp. 189-193, 2004.

[6] M. M. Stecker, "Nerve stimulation with an electrode of finite size: differences between constant current and constant voltage stimulation," Computers in Bio. and Med., vol. 34, pp. 51-94, 2004.

[7] M. N. O. Sadiku, Numerical Techniques in Electromagnetics. Boca Raton, FL: CRC Press, 2000, ch. 3.

[8] V. T. Z. Krasteva, S. P. Papazov, and I. K. Daskalov, "Peripheral nerve magnetic stimulation: influence of tissue non-homogeneity,” BioMed. Eng. Online, vol. 2 pp 19, 2003.

[9] D. J. Ford, C. Pither, P. P. Raj, "Comparison of insulated and uninsulated needles for locating peripheral nerves with a peripheral nerve stimulator,” Anesth. Anal., vol. 63 pp. 925-928, 1984.

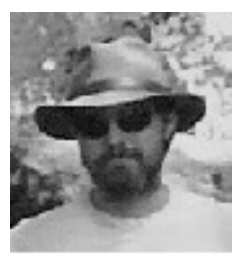

James Ch. Davis started out as a child in the great state of Ohio and moved to Florida when he was young. After high school, he entered the Honors Program at the University of Florida, where he worked in the experimental condensed matter physics group of Mark W. Meisel. After four years of juggling projects, including this one, James graduated with a B.S. degree in Physics and, in Fall 2006, entered the graduate program in physics at The Ohio State University. An avid hiker of remote national trails, James enjoys life and works at not being confused with James C. Davis, a Professor of Physics at Cornell University. 


\section{Figure Captions}

Fig. 1. Side photographs of the Stimuplex (left) and Contiplex (right) needles under a microscope. The Simuplex bevel angle was taken as $30^{\circ}$, while the Contiplex bevel is approximately $15^{0}$. The light areas are covered with Teflon insulation, while the darker region near the Stimuplex needle tip is bare metal. The Contiplex has an exposed conducting surface completely perpendicular to the microscope stage in this image, and therefore it is not visible in this image.

Fig. 2. Frontal photographs of the Stimuplex (left) and Contiplex (right) needles under a microscope. Bare metal surfaces of the Stimuplex needle are the dark areas, while the thin uninsulated areas of the Contiplex needle are indicated by black curves. Inner diameter of the Stimuplex shaft is $0.4 \mathrm{~mm}$, and the Contiplex inner shaft diameter is $1 \mathrm{~mm}$.

Fig. 3. 2-D planar slices of the 3-D electric field around the Stimuplex needle, taken perpendicular the shaft axis (z), with black dots representing an overhead view of all voltage source points modeled. The needle tip is taken as $\mathrm{z}=0$. Inset graphics include a line representing the approximate z-value of the corresponding planar slice. The middle image (b) most closely mimics the field present on the surface of a flat nerve bundle perpendicular to and nearly touching the incoming needle tip.

Fig. 4. 2-D planar slices of the 3-D electric field around the Contiplex needle, taken perpendicular to the shaft axis (z), with black dots representing an overhead view of all voltage source points modeled. The Contiplex profile is seen to be significantly elongated, compared to the more symmetric field of the Stimuplex. This difference is attributable, in part, to variation in the tip angles with respect to the shaft. 
Fig. 5. 2-D planar slices of the 3-D electric field around the Stimuplex (a) and Contiplex (b) needles taken at a $30^{\circ}$ angle to the shaft axis (z), with black dots representing voltage source points as projected onto this plane. The inset graphics illustrate the orientation of each plane. The shifts in field strength and distribution between (a) and (b) are largely reflections of the different bevel angles of each tip.

Fig. 6. View of the electric field distribution for Stimuplex (a) at $-30^{\circ}$ and Contiplex (b) at $-30^{0}$ with respect to the shaft. The inset graphics show the orientation of each plane. Comparing Figs. 5 and 6 illustrates the effects of a $180^{\circ}$ rotation about the needle shaft (z) axis. 
TBME-00604-2005.R3

Fig. 1

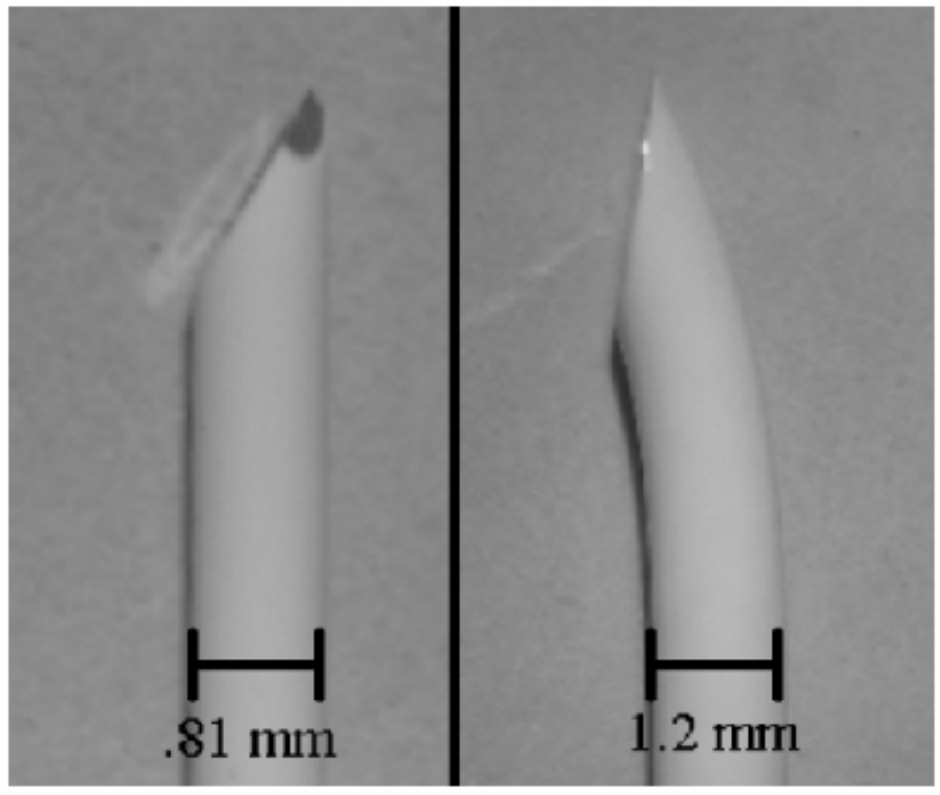


TBME-00604-2005.R3

Fig. 2

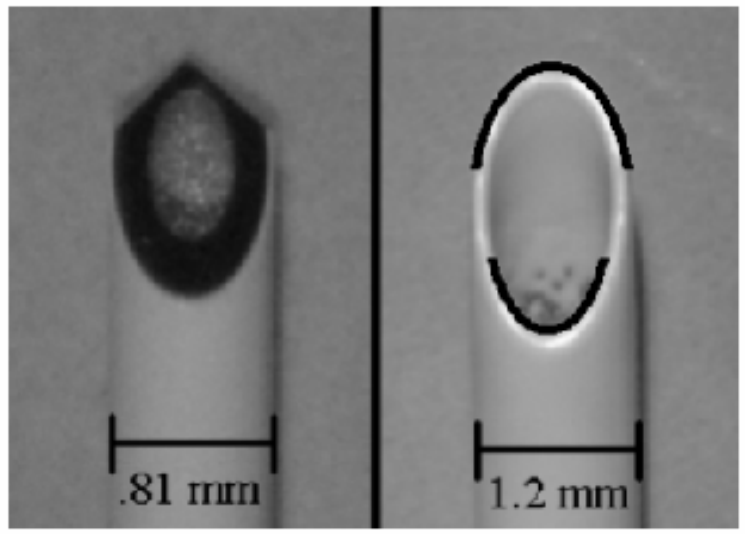


TBME-00604-2005.R3

Fig. 3
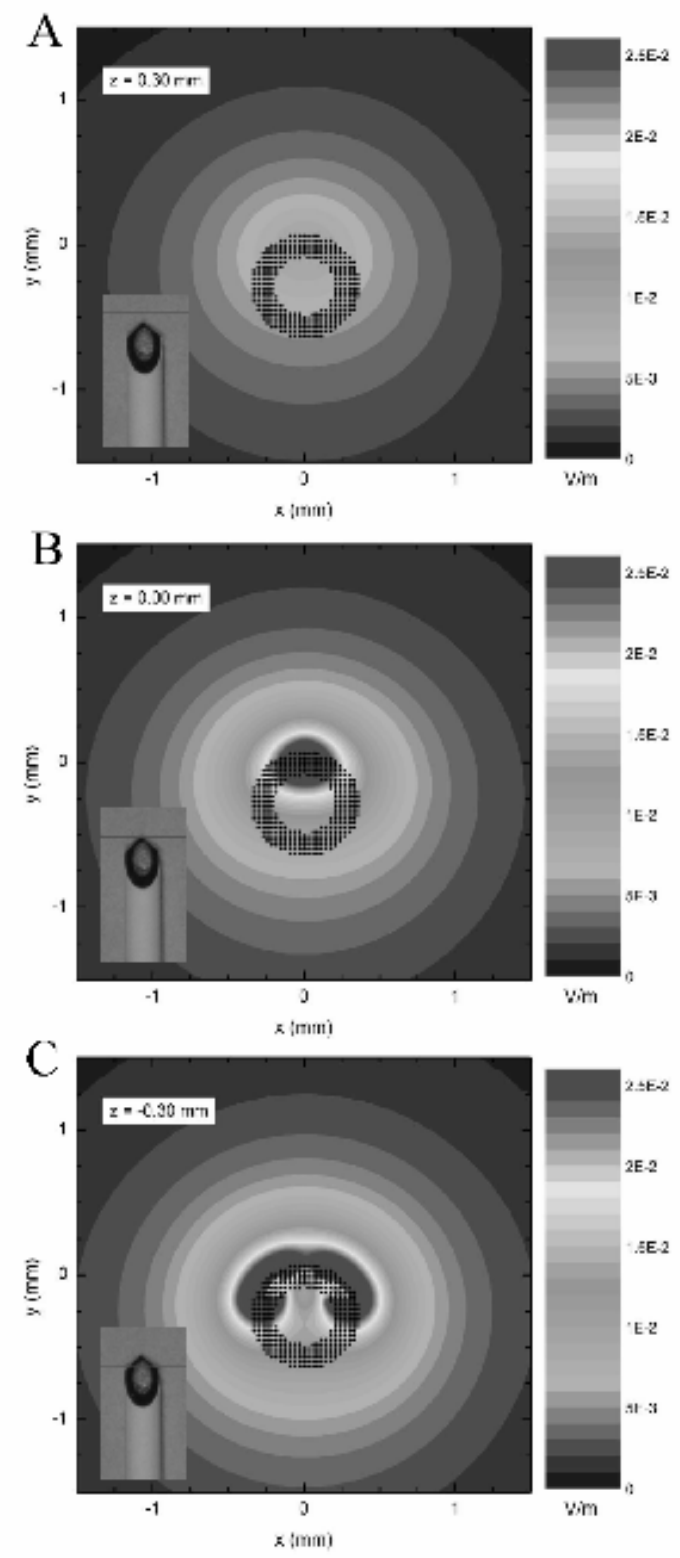
TBME-00604-2005.R3

Fig. 4
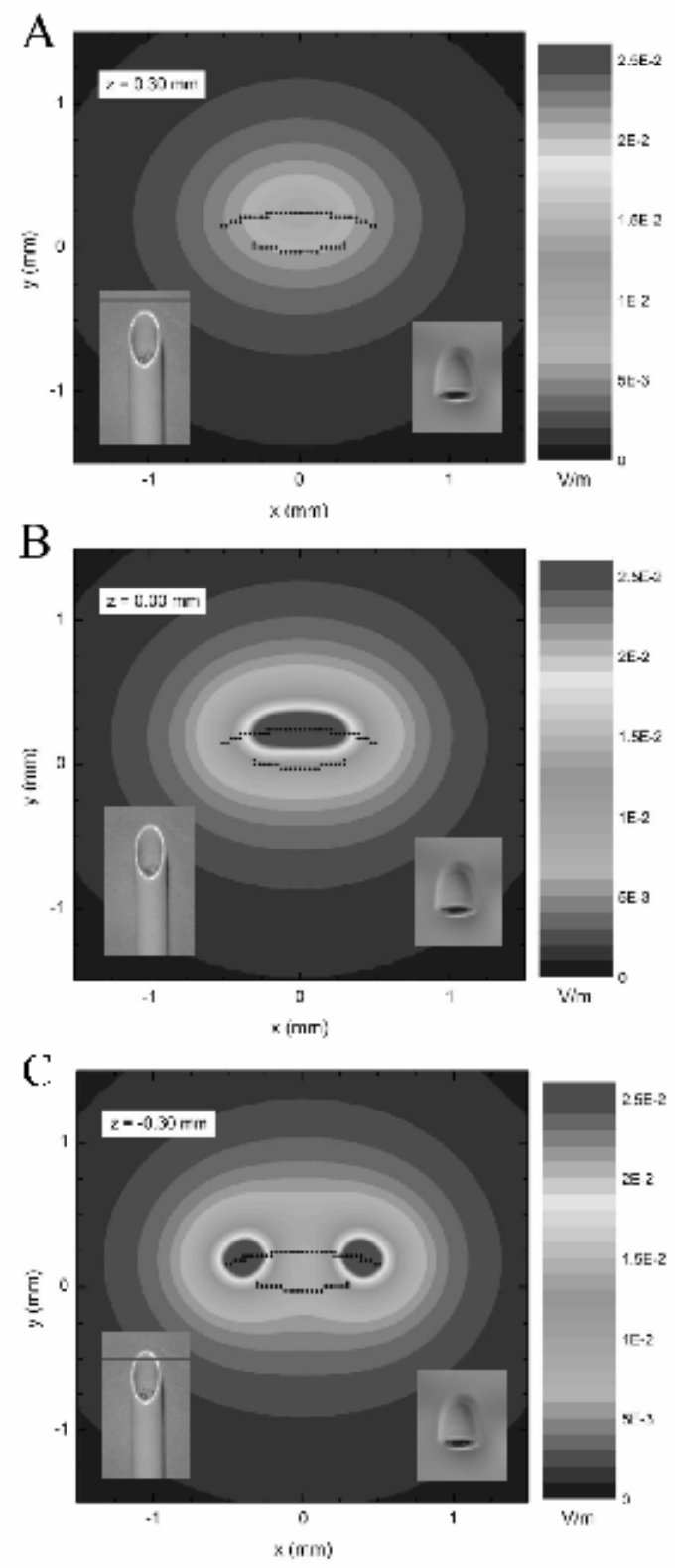
TBME-00604-2005.R3

Fig. 5
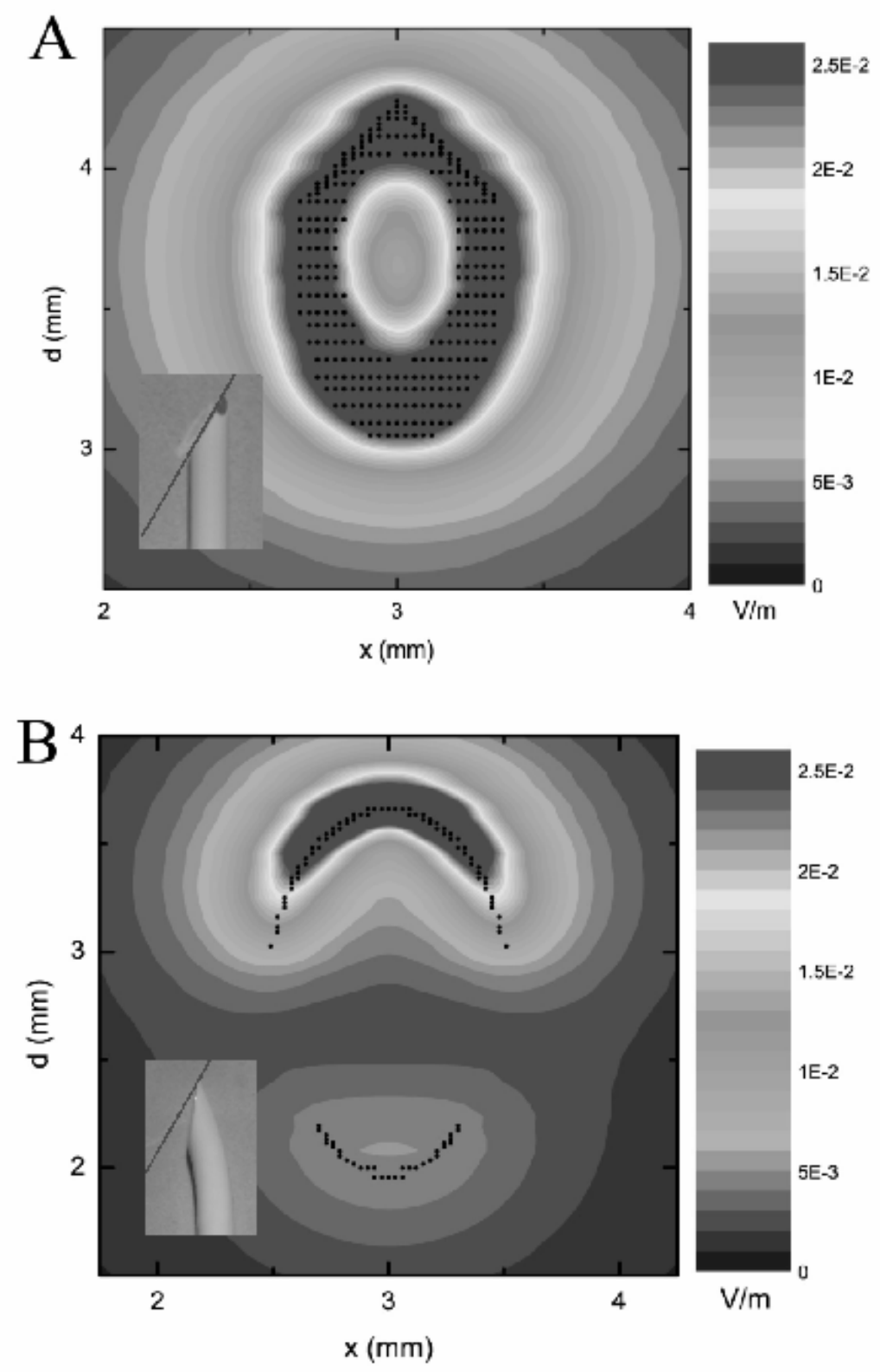
TBME-00604-2005.R3

Fig. 6
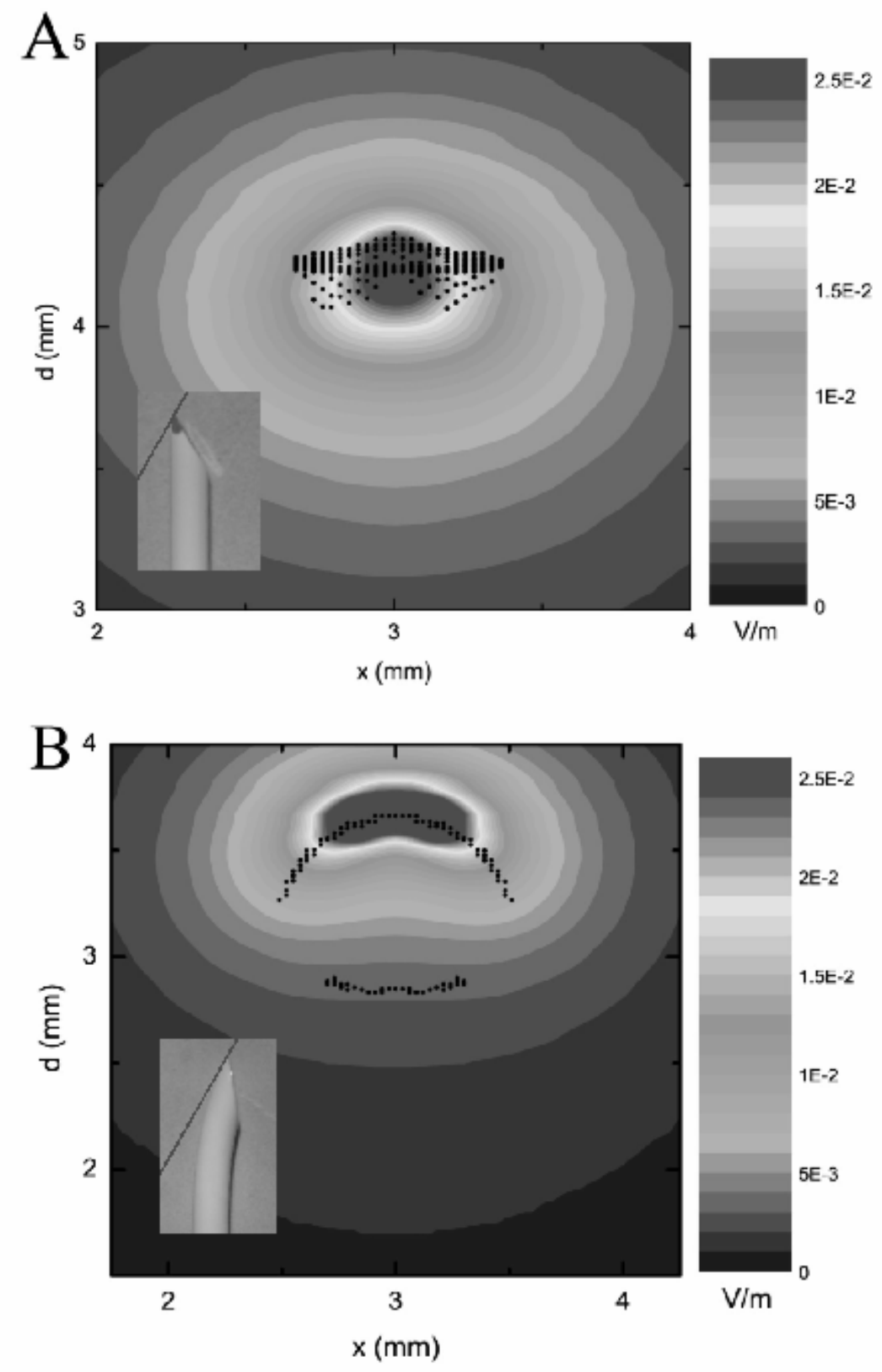